\title{
Systeme zum Ein- oder Austrag von Schütt- gütern in oder aus Druckräumen
}

\author{
Werner Stahl und Reinhold Stadler*
}

In vielen Bereichen der Chemie- und Aufbereitungstechnik werden aus thermodynamischen oder wirtschaftlichen Gründen Prozesse bei Drücken durchgeführt, die. vom Umgebungsdruck abweichen. Sind die Reaktanten und Produkte dieser Prozesse staubförmige oder körnige Feststoffe, so ergeben sich bei deren Ein- bzw. Austrag aus den Bereichen höheren oder niedrigeren Druckes zahlreiche Probleme. Einerseits sollen die Guteigenschaften (z. B. Korngröße, Fließverhalten) so wenig wie möglich verändert werden, andererseits strebt man einen möglichst kontinuierlichen Guttransport zwischen den Räumen unterschiedlichen Druckes an. Weiterhin soll der Gasaustausch gering gehalten werden. Druckschwankungen im Prozeßraum sind weitestgehend auszuschließen. Das Ziel dieser Arbeit war eine zusammenfassende Darstellung aller Systeme, die zum Ein-/Ausschleusen von Schüttgütern in oder aus Räumen, die vom Umgebungsdruck abweichen, geeignet sind. Die einzelnen Schleusen werden für gegebene Anforderungen auch qualitativ beurteilt. Für das Ausschleusen von Filterkuchen aus Überdruckräumen wird eine neue Lösung vorgestellt.

\begin{abstract}
Systems for the charging or discharging of bulk solids into or out of pressure chambers. In many branches of chemical and dressing technology there are compelling thermodynamic or economic reasons for carrying out various processes at pressures deviating from ambient values. If the reactants and products of these processes are dusts or granular solids then numerous problems are encountered on transfer between regions of higher or lower pressure. On the one hand, the properties of the feed (such as particle size, flow behaviour) should undergo the slightest possible modification, and on the other hand the transport of the material between the regions of differing pressure should be as continuous as possible. Moreover, the gas transport should be kept low. Pressure swing in the process region is to be avoided. The aim of this study was to survey all systems suitable for introduction or removal of bulk solids into or out of chambers at pressures other than the ambient value. The various pressure locks are assessed qualitatively for given tasks. A new solution is presented for the discharge of filter cake from highpressure chambers.
\end{abstract}

\section{Einleitung}

Alle verfahrenstechnischen Prozesse, bei denen das Problem der Ein- und Ausschleusung von Schüttgütern in oder aus Räumen unterschiedlichen Druckes auftritt, unterscheiden sich in vielen Umgebungs- und Randbedingungen. Es ist deshalb nicht möglich, ein für nahezu alle Anforderungen gleich gut geeignetes Schleussystem zu finden. Tab. 1 zeigt einen groben Überblick über die Anwendungsgebiete, in denen bei sehr unterschiedlichen Bedingungen heute bereits Ein-/Austragssysteme gefordert werden und nur zum Teil technisch realisiert sind.

Schleussysteme sind ferner in der Zukunft für die folgenden Prozesse denkbar, die heute teils diskontinuierlich, teils unter Normaldruck ablaufen:

- Ein- und Austrag von Katalysatorschüttungen,

- Mischen von Feststoffen und Weiterverarbeitung unter Druck,

- Zwischenspeichern von Schüttgütern unter Druck,

- Calcinierprozesse,

- Drucktrockner.

Eine Erhöhung des Druckes und ein kontinuierlicher Verfahrensablauf würden die Prozesse wirtschaftlicher gestalten.

\section{Methodische Analyse der Schleussysteme}

\subsection{Funktionsstruktur}

Die Gesamtfunktion „,Schüttgüter in und aus Druckräumen ein- und austragen "wird soweit in Teilfunktionen aufgelöst, bis eine Lösungssuche für die betreffenden Teilfunktionen erfolgreich erscheint. Zur Erstellung der ,Allgemeinen
Funktionsstruktur" wird eine Differenzierung der bekannten Systeme nach ihren physikalischen Wirkprinzipien vorgenommen (Vorgehensweise nach [7]).

Die allgemeine Funktionsstruktur (Abb. 1) gilt demnach für alle bekannten Systeme gleichermaßen. Sie setzt sich aus Energie-, Stoff- und Signalfluß zusammen. Die aufgeführten Teilfunktionen sind entsprechend ihrer Grundfunktionen Führen $(\mathrm{F})$, Trennen $(\mathrm{T})$, Speichern $(\mathrm{S})$ bezeichnet und numeriert. Die einzelnen Teilfunktionen sind häufig in ihrer Reihenfolge vertauschbar, ohne die Gesamtfunktion zu beeinflussen.

Die gestrichelt umrandeten Teilfunktionen sind wünschenswerte Ergänzungen und zählen nicht zu den Forderungen. Die einzelnen Teilfunktionen werden durch verschiedene konstruktive Elemente erfüllt, die sich in den physikalischen Wirkprinzipien unterscheiden.

\subsection{Physikalische Wirkprinzipien der Ein-/Austragssysteme}

Alle Konzeptvarianten werden zunächst nach den physikalischen Wirkprinzipien der Abdichtung eingeteilt in:

$-(\alpha)$ Systeme, in denen das Schüttgut diskontinuierlich (chargenweise) oder quasi-k ontinuierlich in Kammern eingefüllt und dort auf ein anderes Druckniveau gebracht wird. Dabei erfolgt die Abdichtung nicht über das Schüttgut, sondern über zusätzliche mechanische Einrichtungen. Der Feststoff wirkt folglich nicht aktiv an der Druckänderung mit, sondern wird in der Schleuse lediglich be- oder entgast.

\footnotetext{
* Prof. Dr.-Ing. W. Stahl und Dipl.-Ing. R. Stadler, Institut für Mech. Verfahrenstechnik und Mechanik, Univ. Karlsruhe, Postfach 6380,7500 Karlsruhe 1.
} 
Tabelle 1. Beispiele für die Anwendungsbereiche von Ein-/Austragssystemen.

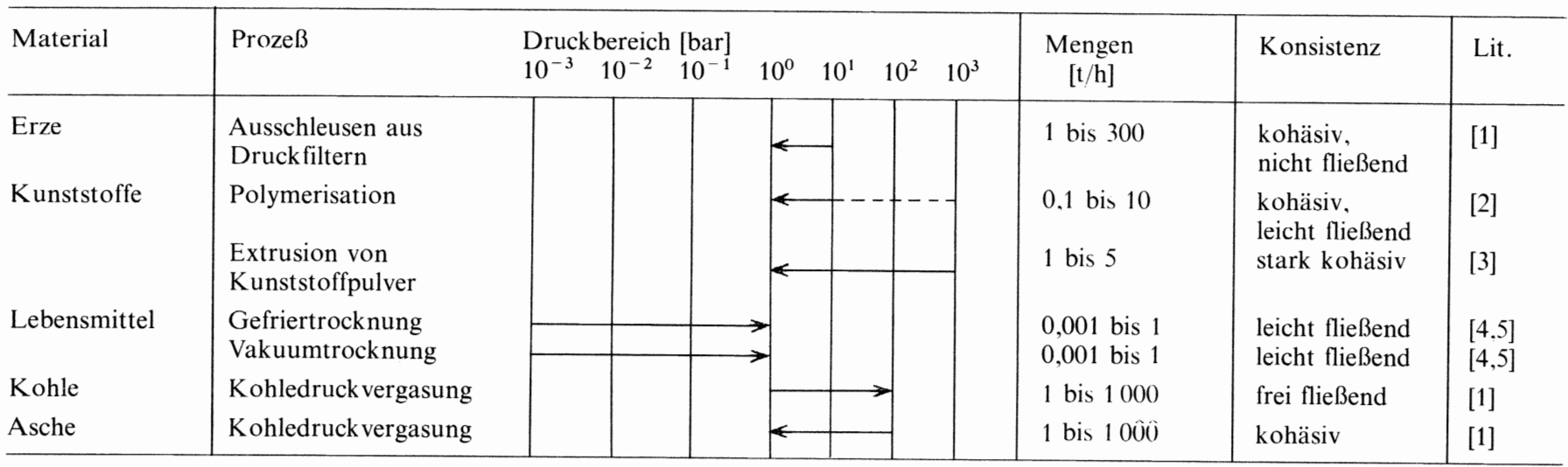

Es ist allerdings unbekannt, ob und wie sich der Packungszustand bei Be- oder Entgasung vor allem bei großen Druckdifferenzen verändert.

Die zeitliche oder örtliche Druckänderung wird über die konstruktive Ausführung vorgegeben oder gesteuert. Die Leckraten sind dabei durch den apparativen Aufbau und das Gut eindeutig festgelegt.

Beispiele: - Kammerschleusen,

- Verdrängersysteme,

- Zellenradschleusen,

- Gliederkettenförderer.

- ( $\beta$ ) Apparate, in denen das Schüttgut kontinuierlich oder quasi-kontinuierlich auf ein anderes Druckniveau überführt wird. Dabei erfolgt der Druckabbau über die Durchströmung des porösen Haufwerks. Da das Schüttgut hierbei einen monotonen Druckanstieg bzw. -abfall erfährt, können sich dabei Dispersitätszustand, Packungsstruktur, Fließeigenschaften und Durchströmungsverhalten verändern. Im

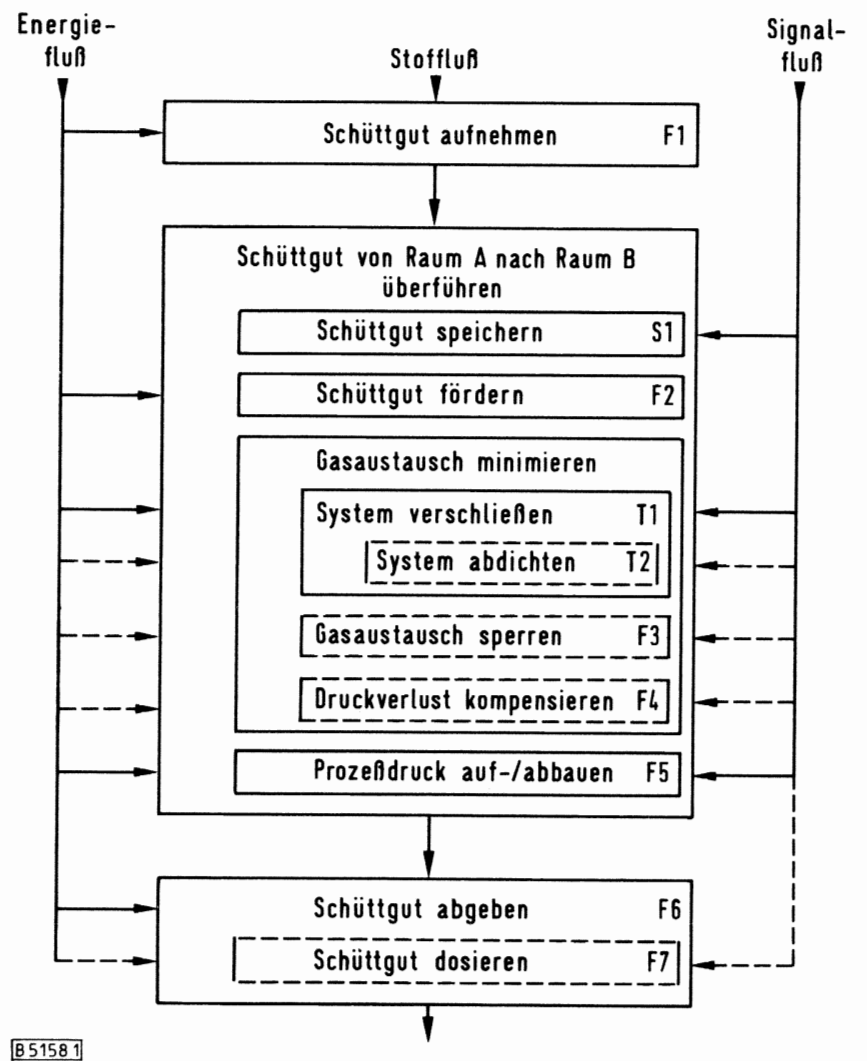

Abb. 1. Allgemeine Funktionsstruktur. einfachsten Fall kann der Transport des Gutes als Festkörperbewegung mit Reibung an der Apparatewand aufgefaßt werden, dem eine Gasströmung überlagert ist. Dadurch entsteht ein „Selbstdichteffekt“ des Gutes. Die Packungsstruktur und ihr Verlauf über der Förderstrecke, ihre Verteilung über den Förderquerschnitt und die konstruktive Ausführung des Apparates sind die wichtigsten Größen, die den Selbstdichteffekt und die Leckgasverluste beeinflussen.

Beispiele: - Gassperrstrecke,

- Produktsäule mit Zentrifugaleintrag,

- Stopfschnecke,

- Kolbenförderer.

- $(\gamma)$ Dynamische Systeme, bei denen durch verschiedene konstruktive Lösungen das Schüttgut auf eine hohe Geschwindigkeit beschleunigt und dann in ständigem Massenstrom in den Raum höheren Druckes eingeblasen wird. Meist wird dem Feststoff zur Beschleunigung ein Gasstrom überlagert. Diese Systeme sind in Betrieb leckgasfrei, aber beim Anund Abfahren müssen zusätzliche Verschluß- und Abdichteinrichtungen vorhanden sein, da im Gegensatz zu den vorhergenannten Systemen bei Stillstand des Apparates keine Abdichtung zwischen den Räumen unterschiedlichen Druckes möglich ist.

Beispiele: - Injektor,

- Zentrifugaleintrag, Rotor,

- pneumatische Förderung.

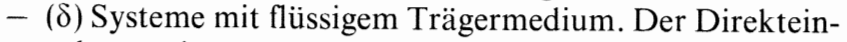
trag des trockenen Feststoffs wird dadurch umgangen, daß das Schüttgut - in einer Trägerflüssigkeit suspendiert eingepumpt und im Druckraum von der Flüssigkeit soweit als möglich wieder abgetrennt wird. Die Trägerflüssigkeit wird anschließend ausgeschleust und rezirkuliert.

Für jedes dieser vier Wirkprinzipien der Abdichtung lassen sich mit Hilfe einer speziellen Funktionsstruktur ,,morphologische Kästen“" erstellen, die eine übersichtliche Anordnung aller Teilfunktionen und deren konstruktiven Elemente darstellen, s. Abb. 2 bis 5. Innerhalb der morphologischen Kästen sind beliebige Kombinationen von Teillösungen möglich, um die Gesamtfunktion zu erfüllen. Einschränkend auf die Zusammenstellung von Einzellösungen wirken die Verträglichkeitsbedingungen, die verschiedene Kombinationen ausschließen.

Die morphologischen Kästen dienen dazu, die bereits bekannten Systeme einzuordnen nach der Reihenfolge der Teilfunktionen und der jeweils dazugehörenden konstruktiven Erfüllung. Auf diese Weise können die bei der Literaturund Patent-Recherche gefundenen Konzeptvarianten dargestellt werden, und es können vor allem neue Varianten gefunden werden. 





In den morphologischen Kästen sind verschiedene konstruktive Elemente gleichzeitig in mehreren Teilfunktionen enthalten, d. h. ein solches Element erfüllt mehrere Teilfunktionen.

\section{Qualitative Beurteilungsgrundlagen}

\subsection{Einordnung der Systeme nach unterschiedli- chen Teillösungen}

Die Systeme werden nach den in den morphologischen Kästen auftretenden Teillösungen differenziert nach:

- (A) Schleusen, die zwei Räume unterschiedlichen Druckes voneinander trennen und die das Schüttgut nur durch Schwerkrafteinwirkung überführen. Diese Systeme sind auf gewisse Schüttguteigenschaften angewiesen, d.h. im allgemeinen kann kohäsives Material in ihnen nur schlecht überführt werden.

A I Kammerschleusen, Dichtprinzip $\alpha$,

A II Verdrängersysteme, Dichtprinzip $\alpha$,

A III Zellenradschleusen, Dichtprinzip $\alpha$,

A IV Gassperrstrecken, Dichtprinzip $\beta$,

A V Produktsäule mit Zentrifugaleintrag, Dichtprinzip $\beta$,

A VI Schleusen zum Eintragen von Schüttgut in pneumatische Förderanlagen (ohne Zellenradschleusen), Dicht prinzip $\alpha, \gamma$,

A VII sonstige Schleussysteme, Dichtprinzip $\alpha, \beta, \gamma$.

- (B) Schleusen, in denen der Materialtransport zwangsweise erfolgt. Diese Systeme sind im allgemeinen nicht auf besondere Schüttguteigenschaften angewiesen.

B I Pneumatische Förderung, Dichtprinzip $\gamma$,

B II hydraulische Förderung, Dichtprinzip $\delta$,

B III Stopfschnecke (Extruder), Dichtprinzip $\beta$,

B IV Schnecke mit Zentrifugaleintrag, Dichtprinzip $\beta$,

B V Kolbenförderer, Dichtprinzip $\beta$,

B VI Gliederkettenförderer, Dichtprinzip $\alpha$,

B VII Schüttgutpumpen, Dichtprinzip $\alpha, \beta$,

B VIII sonstige Schleussysteme, Dichtprinzip $\alpha, \beta$.

\subsection{Einordnung der Systeme nach der Anforderungsliste}

Alle Schleussysteme können entsprechend ihrer Eignung nach den wichtigsten Punkten einer allgemein gültigen Anforderungsliste nach folgendem Schema eingeordnet werden:

a) Richtung der Gasströmung:

1) Gasströmung sowohl in Richtung des Guttransportes als auch entgegengesetzt möglich;

2) Gasströmung in Richtung des Guttransportes, d.h. Ausschleusen aus Überdruckräumen oder Einschleusen in Unterdruckräume;

3) Gasströmung entgegen der Richtung des Guttransportes, d. h. Ausschleusen aus Unterdruckräumen oder Einschleusen in Überdruckräume.

b) Differenzdruck bereich:
1) $\frac{p_{\max }}{p_{\min }}>5$;
2) $\frac{p_{\max }}{p_{\min }} \leqslant 5$

c) Überführung des Schüttgutes: Der Feststoff wird

1) kontinuierlich,

2) quasi-kontinuierlich,

3) diskontinuierlich

ein- bzw. ausgeschleust.

d) Beanspruchung des Schüttgutes: Während des Guttransportes erfolgt
1) keine Kornbeanspruchung und keine Kompaktierung,

2) Kornbeanspruchung aber keine Kompaktierung,

3) Kornbeanspruchung und Kompaktierung.

e) Sperrung des Gasaustausches: Falls erforderlich, kann der Gasaustausch zwischen den Bereichen verschiedenen Druckes vollständig gesperrt werden

1) ja; 2) nein.

f) Arbeitstemperaturbereich: Das System ist geeignet für

1) alle Temperaturen,

2) $-20^{\circ} \mathrm{C}<\vartheta<150^{\circ} \mathrm{C}$,

3) $\vartheta \geqslant 150$ C.

Da die Schleusen durch geeignete Werkstoffauswahl ihrer Arbeitstemperatur angepaßt werden können, werden in diesem Punkt nur eventuell vorhandene Dichtungen und ihre Temperaturabhängigkeit betrachtet.

g) Korngrößenbereich [1]: Mit dem Schleussystem können Schüttgüter, die aus

1) allen Korngrößen,

2) Feinstgut ( 0 bis $0,5 \mathrm{~mm})$,

3) Feingut (0 bis $10 \mathrm{~mm}$ ),

4) Grobgut (10 bis $60 \mathrm{~mm}$ )

bestehen, überführt werden.

h) Abrasivität des Schüttgutes:
1) stark schleißendes,
2) leicht schleißendes,
3) nicht schleißendes

Material kann mit der Schleuse zwischen den Druckbereichen transportiert werden.

i) Fließverhalten: Das System eignet sich für

1) stark kohäsives,

2) leicht kohäsives,

3) frei fließendes

Schüttgut.

j) Abhängigkeit des Leckgasstromes vom Durchsatz: Eine Durchsatzänderung beeinflußt den Leckgasstrom

1) nein; 2) ja.

k) Allgemeine Forderungen an das Konzept des Systems: Einfache Herstellung; kleine Baugrößen; geringes Gewicht; hoher Durchsatz; einsetzbar in jeder räumlichen Lage; einfache Bedienung; einfache Konstruktion; wenig Bauteile; preisgünstig; geringer Energieverbrauch;

1) mindestens fünf Anforderungen,

2) weniger als fünf Anforderungen sind erfüllt.

1) Allgemeine Forderungen an die Funktionserfüllung: Keine Konsistenzänderung; keine Korngrößenänderung; Vermeiden von Druckschwankungen im Prozeßraum; einfache und sichere Abführung des Schleusgases; hoher Füllgrad ; kleine Spaltverluste; Güte der Abdichtung; Regelbarkeit des Durchsatzes;

1) vier und mehr Anforderungen,

2) weniger als vier Anforderungen sind erfüllt.

\subsection{Kennzeichnung der Schleussysteme}

Die Einordnung der Systeme erfolgt nach unterschiedlichen Teillösungen (Abschnitt 3.1) mit einer Buchstaben-ZahlenKombination und Charakterisierung der Schleusen entsprechend der Erfüllung ihrer Anforderungen nach der Liste aus Abschnitt 3.2 mit einer zwölfstelligen Zahlenreihe in der Reihenfolge a) bis 1). Die Wahl der Reihenfolge der Anforderungen in den einzelnen Punkten der Abschnitte a) bis 1) erfolgte nach den Einsatzbereichen. Kleine Zahlen (1) bedeuten sehr umfangreiche Einsatzmöglichkeiten, während die mit $(2,3,4)$ gekennzeichneten Anforderungspunkte in der Regel einen geringeren Erfüllungsgrad signalisieren. 
Tabelle 2.

Charakterisierung der Schleusen.

\begin{tabular}{|c|c|c|c|}
\hline Schleusenbauart & & Klassifizierung & $\begin{array}{l}\text { Mittel- } \\
\text { wert } \bar{x}\end{array}$ \\
\hline Kammerschleusen & A I & $1 / 1-2 / 3 / 1 / 1 / 1 / 1 / 1-2 / 2-3 / 1 / 2 / 1$ & 1,46 \\
\hline $\begin{array}{l}\text { Verdränger- } \\
\text { systeme }\end{array}$ & A II & $1 / 1-2 / 3 / 1-2 / 1 / 1 / 1 / 1-2 / 2 / 1 / 2 / 1$ & 1,46 \\
\hline $\begin{array}{l}\text { Zellenrad- } \\
\text { schleusen }\end{array}$ & A III & $1 / 2 / 2 / 1-2 / 2 / 2 / 3 / 2 / 2-3 / 1 / 1 / 2$ & 1,83 \\
\hline $\begin{array}{l}\text { Gassperrstrecken } \\
\text { ohne Gutverdich- } \\
\text { tung }\end{array}$ & A IV & $3 / 2 / 1 / 1 / 2 / 1 / 2-3 / 1 / 3 / 2 / 1 / 2$ & 1,79 \\
\hline $\begin{array}{l}\text { Gassperrstrecken } \\
\text { mit Gutverdich- } \\
\text { tung }\end{array}$ & A IV & $1 / 1-2 / 1 / 1-2 / 2 / 1 / 2-3 / 1 / 2 / 1-2 / 1 / 2$ & 1,50 \\
\hline $\begin{array}{l}\text { Produktsäulen mit } \\
\text { Zentrifugaleintrag }\end{array}$ & A V & $3 / 1 / 1 / 1-2 / 1 / 1 / 2-3 / 1 / 1-2 / 1 / 1 / 2$ & 1,46 \\
\hline $\begin{array}{l}\text { Schleusen zum Ein- } \\
\text { führen von Schütt- } \\
\text { gütern in pneuma- } \\
\text { tische Förder- } \\
\text { anlagen }\end{array}$ & A VI & $3 / 2 / 1-2 / 2 / 2 / 1 / 1 / 1-2 / 2 / 2 / 2 / 1-2$ & 1.72 \\
\hline $\begin{array}{l}\text { sonstige Schleus- } \\
\text { systeme ohne }\end{array}$ & & & 1,12 \\
\hline Fördereinrichtung & keine & Einordnung & \\
\hline $\begin{array}{l}\text { pneumatische } \\
\text { Fördersysteme }\end{array}$ & B I & $2 / 2 / 1-2 / 1 / 2 / 1-3 / 1 / 1 / 2 / 2 / 2$ & 1,67 \\
\hline $\begin{array}{l}\text { hydraulische } \\
\text { Fördersysteme }\end{array}$ & B II & $1 / 1 / 1-2 / 2 / 1 / 2 / 1 / 1 / 1 / 1 / 2 / 1-2$ & 1,33 \\
\hline Schneckensysteme & B III & $1 / 2 / 1 / 2 / 2 / 1 / 1 / 2 / 1 / 2 / 1 / 2$ & 1,58 \\
\hline Extruder & B III & $1 / 1 / 1 / 3 / 1-2 / 1 / 2-3 / 3 / 1 / 1 / 1 / 1$ & 1,50 \\
\hline $\begin{array}{l}\text { Zweiwellen- } \\
\text { extruder }\end{array}$ & B III & $1 / 1 / 1 / 2 / 1 / 1 / 2-3 / 2 / 1 / 1 / 1 / 1$ & 1,30 \\
\hline $\begin{array}{l}\text { Schnecken mit } \\
\text { Zentrifugaleintrag }\end{array}$ & B IV & $3 / 1 / 1 / 2 / 1 / 2-3 / 1-2 / 1 / 1 / 1 / 2$ & 1,50 \\
\hline Kolbenförderer & $\mathrm{B} \mathrm{V}$ & $1 / 1 / 3 / 3 / 1 / 1 / 1 / 3 / 1 / 1 / 1 / 1$ & 1,50 \\
\hline $\begin{array}{l}\text { Gliederketten- } \\
\text { förderer }\end{array}$ & B VI & $1 / 1 / 2 / 1 / 1 / 2 / 1 / 3 / 2 / 2 / 2 / 1$ & 1,58 \\
\hline $\begin{array}{l}\text { Schüttgutpumpen } \\
\text { sonstige Schleus- } \\
\text { systeme mit För- }\end{array}$ & keine & Einordnung & \\
\hline
\end{tabular}

Die Handhabung dieser Kennzeichnung ist einfach, und es ist auf den ersten Blick ersichtlich, ob ein System auf breiter Ebene oder nur für einen speziellen Anwendungsfall eingesetzt werden kann.

Sobald ein System eine geforderte Eigenschaft nicht erfüllen kann, scheidet es aus. Nach diesem Beurteilungssystem können die in Abschnitt 3.1 dargestellten Schleussystemgruppen nach Tab. 2 charakterisiert werden. (Der angegebene Mittelwert ergibt sich bei gleicher Bewertung aller Punkte a) bis 1) und dient nur zur Vorauswahl.)

\section{Berechnungsgrundlagen}

Zur Dimensionierung einer Schleuse und auch bereits zur Bewertung sollte das Verhalten des Materials in der Schleuse bekannt sein.

Neben Anforderungen an die Qualität und Verarbeitbarkeit von Werkstoffen für den Bau der Schleuse wurde die Literatur zu folgenden Punkten ausgewertet:

- Strömung durch Packungen [8, 9, 32, u. a.],

- Fließkriterien für Packungen [13, 14, u. a.].

Im weiteren soll auf die ausführliche Fassung dieses Beitrages [33] verwiesen werden.

\section{Darstellung verschiedener Schleussysteme}

Beispielhaft werden zu den wichtigsten der 15 Gruppen aus Abschnitt 3.1 einige Varianten dargestellt. Von einer weiteren Varianten-Entwicklung nach dem morphologischen Kasten, vor allem von Kombinationen der einzelnen Hauptgruppen, wird in dieser Arbeit abgesehen. (In den morphologischen Kästen sind ca. 4,5 Mio. sinnvolle Varianten enthalten.) Dem Benutzer wird vielmehr die Möglichkeit gegeben, mit diesem Rüstzeug das für ihn optimale oder wenigstens das seinen Wünschen am meisten entgegenkommende System auszuwählen und zu optimieren.

\subsection{Kammerschleusen}

Die Kammerschleuse ist das zur Zeit am häufigsten verwendete Ein-/Austragssystem. Kammerschleusen können bis zu Temperaturen von $400^{\circ} \mathrm{C}$, Drücken von max. 100 bar sowie Durchsätzen von max. $70 \mathrm{t} / \mathrm{h}$ zuverlässig eingesetzt werden [15]. Nach Auffüllen der Schleuse mit Gut erfolgt

- beim Einschleusen in Druckräume eine Bespannung mit Prozeß- bzw. Fremdgas bis auf Prozeßdruck und nach dem Einschleusen eine Entspannung der Schleusenkammer, wobei das Entspannungsgas nach erneuter Verdichtung wieder dem Prozeß zugeführt werden kann. Bei der Bespannung der Schleuse mit Prozeßgas muß darauf geachtet werden, daß das Gut bei der Kondensation des schlagartig entspannten Prozeßgases nicht zu sehr befeuchtet wird,

- beim Ausschleusen aus Druckräumen zunächst eine Entspannung (evtl. mit Schleusengas-Rückführung) und nach dem Austrag eine erneute Bespannung der Schleuse. Um den Hauptnachteil der Kammerschleuse, den diskontinuierlichen Ablauf des Schleusvorganges, auszugleichen, wurde die Zwillingsschleuse entwickelt (Abb. 6). Die beiden

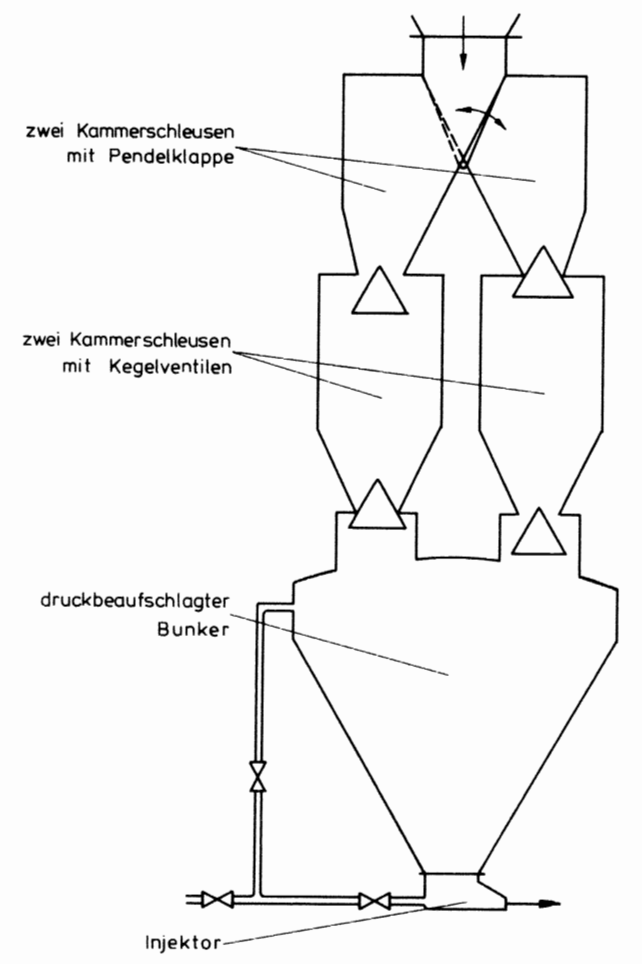

Abb. 6. Mehrkammer-Schleussystem zur pneumatischen Förderung [16]; Einordnung: A Ia 3/1/2/1/1/1/3/2/2/1/2/1. 
Kammerschleusen werden abwechselnd gefüllt und entleert. Durch einen Druckausgleich zwischen beiden Behältern kann der Schleusgasverlust um 40\% reduziert werden. Ein weiterer Vorteil besteht darin, daß bei Ausfall eines Ventils die Schleuse noch als Einkammerschleuse funktionsfähig ist.

Nachteile der Kammerschleuse sind:

- die diskontinuierliche Arbeitsweise und

- Schleusengasverlust,

- Druckschwankungen im Prozeß beim Öffnen der Kammerschleuse durch das große Kammervolumen,

- kohäsive Produkte können ohne zusätzliche Einrichtungen schlecht ausgetragen werden.

Vorteile sind:

- sehr hohe Differenzdrücke können überwunden werden und

- die beiden Prozeßräume können voneinander völlig unabhängig sein, da die Schleuse be- oder entgast werden kann,

- nahezu alle Produkte, vor allem alle Korngrößen, können verarbeitet werden,

- die Kammerschleusen sind bereits bewährte Systeme, über deren Einsatzmöglichkeiten und Auslegung große Erfahrung vorliegt,

- die Guteigenschaften werden nicht verändert.

\subsection{Verdrängersysteme}

Die Systeme bestehen im allgemeinen aus einem zylindrischen Gutaufnahmebehälter und einem darin beweglichen Kolben, der das Schleusengas verdrängt (Abb. 7). Verdrän-

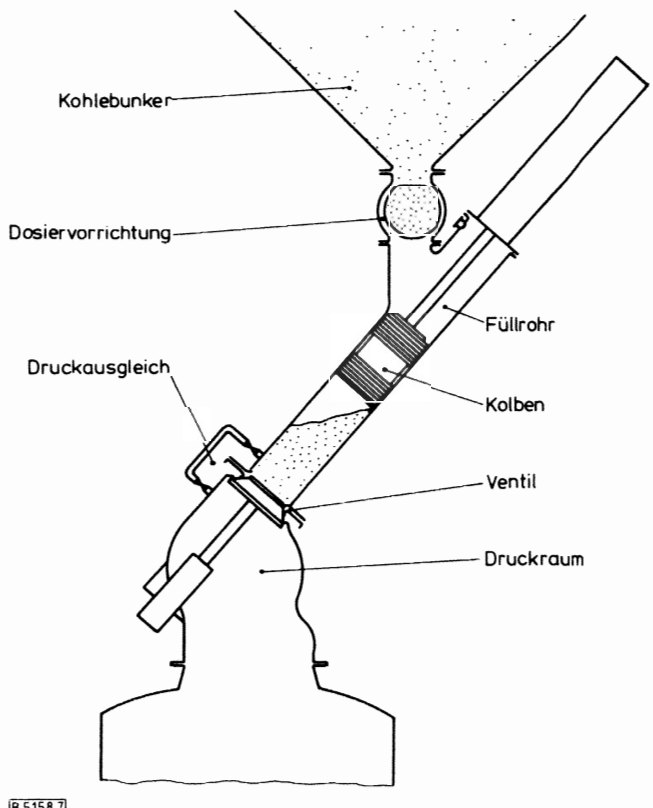

B51587

Abb. 7. Schleuse mit Verdrängerkolben [17]; Einordnung: A II $3 / 1 / 3 / 1 / 1 / 2 / 3 / 2 / 2 / 1 / 2 / 1$.

gersysteme können bis zu Drücken von max. 100 bar und bis zu Durchsätzen von ca. $20 \mathrm{t} / \mathrm{h}$ eingesetzt werden. Die Temperatureinsatzgrenze ist abhängig von der Art der verwendeten Abdichtungen.

Vorteile der Verdrängersysteme sind:

- praktisch kein Schleusengasverlust,

- einfache Rückführung des Gases, das aus dem Raum höheren Druckes eintritt,

- alle Korngrößen sind einsetzbar,

- konstruktiv recht einfache Lösungen,

- keine Änderung der Guteigenschaften,
- hohe Drücke sind zulässig,

- das Gut darf (leicht) kohäsiv sein, da es durch den Verdrängerkolben mit ausgetragen wird.

Nachteile sind:

- der hohe Verschleiß, der auftritt, wenn schleißende Partikeln zwischen Schleuskammer und Verdränger eindringen,

- die diskontinuierliche Arbeitsweise und

- das Problem, daß bewegte Dichtungen auftreten,

- Technologie im Entwicklungsstadium.

\subsection{Zellenradschleusen}

Die Zellenradschleuse (Abb. 8) hat sich zum Ein- und Austrag von Schüttgütern bei geringen Druckdifferenzen zwischen den beiden Druckräumen bewährt. Ihr Haupteinsatz-

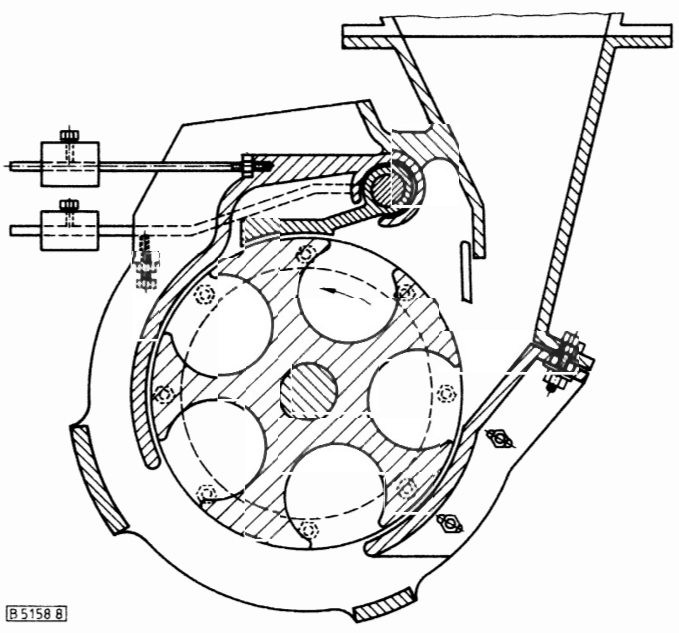

Abb. 8. Zellenradschleuse [18]; Einordnung: A III $1 / 2 / 2 / 1-2 /$ $1 / 2 / 3 / 2 / 2-3 / 1 / 1 / 2$.

gebiet ist das Vergleichmäßigen des Gutstromes und dessen Dosierung, die durch eine Drehzahlregelung einfach möglich ist. Zellenradschleusen werden bei beliebigen Temperaturen eingesetzt bis zu Druckdifferenzen von max. 1,5 bis 3 bar mit Durchsätzen von max. $200 \mathrm{~m}^{3} / \mathrm{h}$.

Vorteile einer Zellenradschleuse sind:

- einfache Dosierung,

- einfache Konstruktion und

- quasi-kontinuierliche Überführung des Gutes.

Nachteile sind:

- der starke Verschleiß, der auftritt, da das Gut an den Gehäusewänden entlangbewegt wird und

- der große Gasdurchsatz mit dem daraus resultierenden Druckverlust, der sich zusammensetzt aus den Kammerverlusten (d.h. dem Gas, das in die Schleusenkammern eindringt) und den Spaltverlusten (d.h. dem Gas, das durch die Spalte an den Stirnwänden und am Umfang des Zellenrades tritt).

\subsection{Gassperrstrecken}

Für geringe Druckdifferenzen von einigen mbar werden sehr häufig sog. „Gassperrstrecken“ eingesetzt. Hierzu durchfließt Feststoff unter Schwerkrafteinwirkung senkrechte oder geneigte Rohre [19-23, u. a.] (Abb. 9, 10, 11).

Die Dichtwirkung beim Befüllen eines Überdruckraumes resultiert aus dem statischen Druck der Feststoffsäule, dem Reibungsdruck verlust des Gases an der nach unten bewegten Feststoffsäule und dem im Schüttgut eingeschlossenen Gas- 
volumen. Eine Verbesserung der Dichtung soll nach [24] durch Einfüllen von Metallkugeln erreicht werden, zwischen denen sich dann der Feststoff befindet. Mit dieser Vorrichtung wird der statische Druck der Feststoffsäule auf bis zu 20 bar erhöht. Im Druckkessel erfolgt eine Abtrennung der Kugeln, die anschließend in das Rohr zurückgeführt werden.

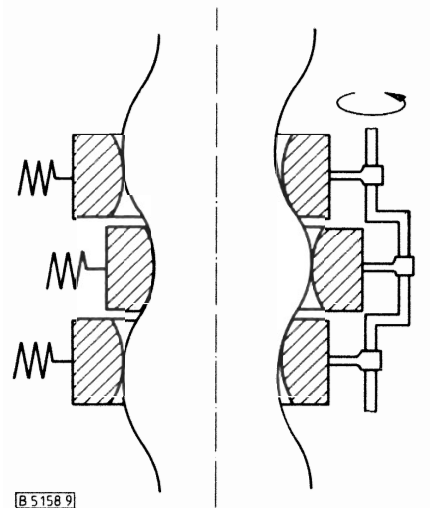

Abb. 9. Feststoffsäule in einem Schlauch mit Beeinflussung des Selbsthemm-Effektes. Der Schlauch hat die Funktionen ,niedriger Randreibwert" und „Abdichtung gegen den Innendruck". Die Radialspannungen werden nicht vom Schlauch aufgenommen, sondern der Schlauch stützt sich in den einzelnen beweglichen Metallringen ab (Inst. für MVM, Univ. K'he 1982); Einordnung: A IV 1/1-2/ $1 / 1 / 2 / 1 / 2-3 / 1 / 1-2 / 1-2 / 1 / 2$.

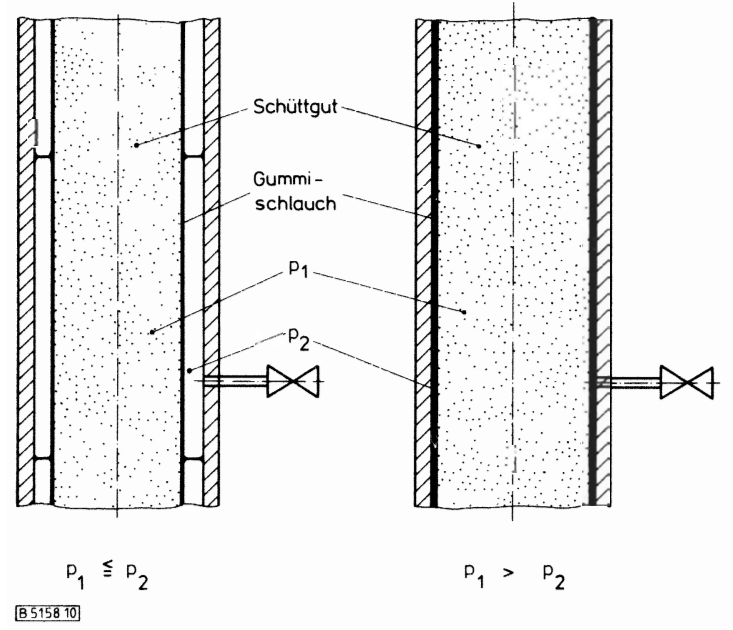

Abb. 10. Rohr mit veränderlichem Durchmesser zur Verdichtung der Feststoffsäule und Steuerung des radialen Haltedrucks (Inst. für MVM, Univ. K'he 1982); Einordnung: A IV $1 / 1-2 / 1 / 1-2 / 2 / 1$ / $2-3 / 2 / 2 / 1-2 / 1 / 2$.

Mit Gassperrstrecken sind Feststoffströme bis zu mehreren $1000 \mathrm{t} / \mathrm{h}$ möglich, bei Temperaturen teilweise über $500 \mathrm{C}$. Der Regelbereich ist im Gegensatz zu anderen Systemen auf den Faktor 5 beschränkt.

Gassperrstrecken können höhere Differenzdrücke überwinden, wenn das Gut in der Feststoffsäule durch Verdichten leicht kompaktiert wird und das Rohr so gestaltet ist, daß durch Beeinflussung des Reibkoeffizienten mit der Wand ein Wiederanfließen gewährleistet wird. Durch diese Beeinflussungsmöglichkeit steckt in der Verbesserung der Gassperrstrecke noch ein weites Entwicklungspotential.

Nachteile von Gassperrstrecken sind:

- Differenzdruck-Schwankungen sowie Änderungen der Schüttguteigenschaften können zu Betriebsstörungen führen,

- es können sich Feststoffbrücken bilden und
- die anlegbare Druckdifferenz zwischen beiden Räumen ist durch die Länge der Sperrstrecke und durch die Schüttdichte bestimmt.

Vorteile sind:

- sehr einfacher Aufbau und

- voll kontinuierlicher Transport,

- für alle Schüttgüter geeignet, wenn durch konstruktive Maßnahmen am Rohr ein Wiederanfließen nach Brükkenbildung im Gut gewährleistet wird,

- alle Korngrößen sind schleusbar.
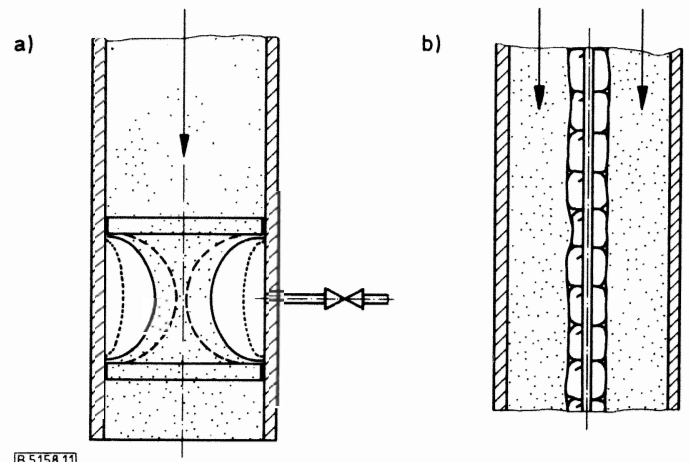

Abb. 11. Verdichtungs- und Zwangstransport-Einrichtung in einer Gassperrstrecke. a) Außenmembran, b) Innenmembran (Inst. für MVM, Univ. K'he 1982); Einordnung: A IV 1/1 -2/1/1 -2/2/1/ $2-3 / 1 / 2 / 1-2 / 1 / 2$.

\subsection{Fliehkraftförderer}

Eine Weiterent wicklung der Gassperrstrecke ist die Produktsäule mit Zentrifugaleintrag, bei der die Gutpartikeln durch Fliehkraft auf hohe Geschwindigkeit beschleunigt werden, wodurch die Gasströmung entgegen der Gutrichtung durch die Reibung an den Partikeln stärker abgebremst wird. Bei diesem dynamischen System wird die überwindbare Druckdifferenz durch den Rotor (= Radialverdichter) erhöht [24-26].

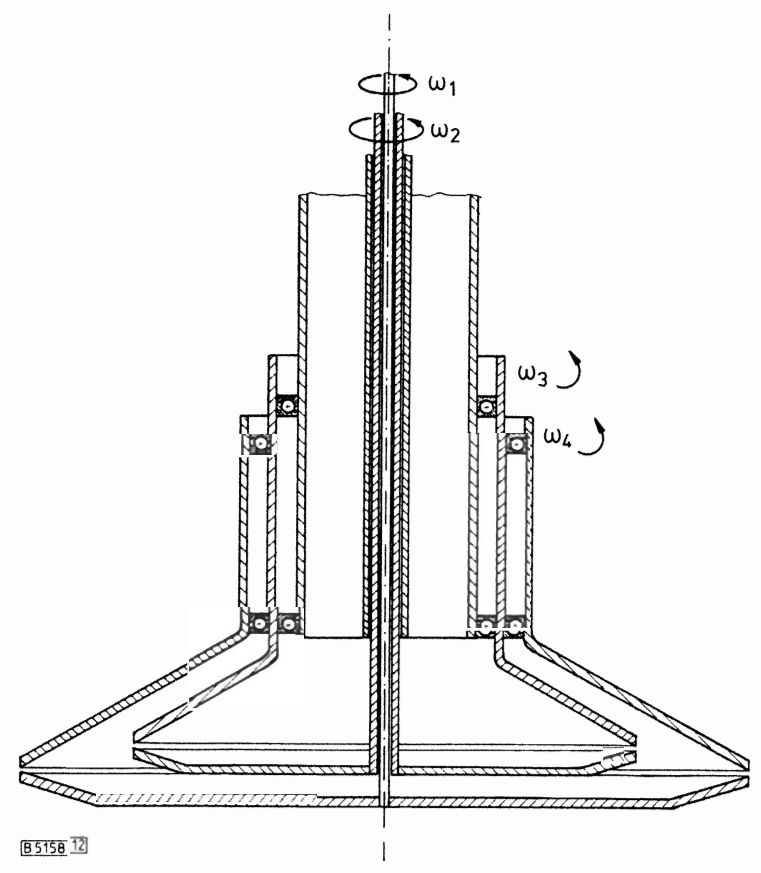

Abb. 12. Erhöhung der Dichtwirkung durch größere Verweilzeit und Steuermöglichkeit (Inst. für MVM, Univ. K'he 1982); Einordnung: A V 3/1/1/1/1/1/2-3/1/1-2/1/1/2. 
Folgende Variante hat sich im Einsatz bei Fest/FlüssigSystemen bereits bewährt: Ein tellerförmiger Rotor kann zum Beispiel mit einer federnden Scheibe verschlossen werden, welche gegen Druck abdichtet. Hat sich eine bestimmte Feststoffmenge vor der Öffnung angestaut, so wird bei entsprechender Drehzahl Material freigegeben. Bisherige Versuchsrotoren arbeiten bei beliebigen Temperaturen bis zu etwa 15 bar gasdicht. In Entwicklung sind Rotoren, die bis zu 100 bar gasdicht sind und die einen Durchsatz von ca. $1 \mathrm{t} / \mathrm{h}$ ermöglichen (dabei werden Drehzahlen bis zu $3000 \mathrm{~min}^{-1}$ erreicht).

In den neueren Entwicklungen werden zum Teil Rotoren verwendet, die aus einer Trag- und einer Deckscheibe bestehen (Abb. 12). Zwischen diesen bildet sich ein kleiner Spalt, der frei von Einbauten ist. Mit dieser Anordnung können jedoch nur geringe Druckdifferenzen $p_{\text {groß }} / p_{\text {klein }}<2$ überwunden werden.

Vorteile des dynamischen Systems sind:

- kontinuierliche Arbeitsweise und

- einfacher Aufbau.

Nachteile sind:

- die meisten dynamischen Systeme (auch die Gassperrstrecke) sind nicht eigensicher, d. h. zum An- oder Abfahren des Systems sind Absperreinrichtungen erforderlich, - die Systeme können nur feine Körnungen verarbeiten.

\subsection{Pneumatische Fördersysteme}

Pneumatische Fördersysteme bestehen grundsätzlich aus zwei Hauptbauteilen, der Luftverdichtungsanlage und einer Gutzufuhr-Einrichtung, die das Schüttgut in die Förderleitung einbringt (Abb. 13). Systeme dieser Art basieren auf dem Wirkprinzip der dynamischen Dichtung. Pneumatische Fördersysteme sind nach $M$. Weber [27] u. a. recht genau auszulegen. Die Systeme sind bis zu 4 bar Druckdifferenz pro Stufe geeignet, die Durchsatzmengen sind vom Verdichter abhängig.

Vorteile der pneumatischen Förderanlage sind:

- recht einfacher Aufbau,

- kontinuierliche Arbeitsweise.

Nachteile dieser Systeme sind:

- der hohe Energieaufwand, der zur Verdichtung des Treibgases, dessen Massenstrom das 0,5- bis 1,5fache des Gutstromes beträgt, erforderlich ist,

- ihre hohe Empfindlichkeit auf Druckschwankungen im angeschlossenen Prozeß,

- Erosions- und Geräuschprobleme, die aufgrund der hohen Strömungsgeschwindigkeiten auftreten,

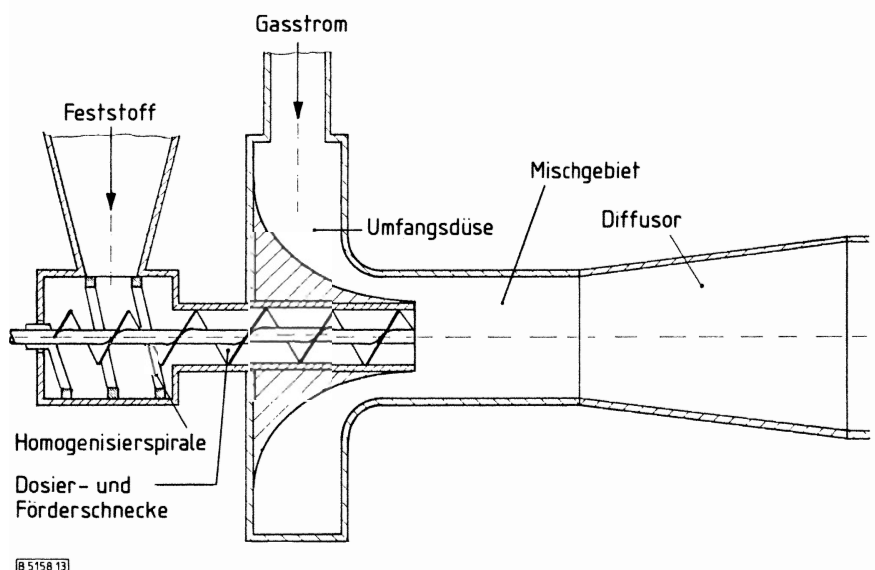

8515810

Abb. 13. Injektor mit zwangsweiser Feststoffzufuhr durch eine Schnecke (Inst. für MVM, Univ. K'he 1981); Einordnung: A VI $3 / 2 / 1 / 2 / 2 / 1 / 2-3 / 2 / 1 / 2 / 2 / 2$.
- zusätzliche Absperrvorrichtungen zum An- und Abfahren sind nötig, da das System nicht selbstdichtend ist.

\subsection{Hydraulische Förderung}

Wie die pneumatische Förderung ist auch die hydraulische Förderung ein dynamisches System, das allerdings Wasser oder Öl als Trägermedium benützt. Dies hat den Vorteil, daß die hydraulische Förderung für wesentlich höhere Drücke als die pneumatische Förderung geeignet ist.

Es existieren verschiedene Arten von Gutzufuhr-Vorrichtungen, wie z. B. Injektoren, bei denen das Gut in einer Düse dem Trägermedium beigemischt wird. Bei einem ZweistufenInjektor, der aus zwei hintereinandergeschalteten Düsen besteht, wird das Gut durch die erste Stufe, eine Hochdruckstufe mit kleinem Durchmesser, beschleunigt, bevor es dann mit hoher Geschwindigkeit in die zweite Düse einströmt, die einen hohen Durchfluß und ein etwas geringeres Druckniveau als die kleine Düse hat. Weiterhin existieren verschiedene Arten von Rohraufgebern, die das Gut über ein Niederdrucksystem, das mit einem Hochdrucksystem über Rohrweichen verbunden ist, ohne Beanspruchung der Hochdruckpumpe aufgeben. Ein weiterer Aufgeber ist das , $\mathrm{Ka}$ myr-System “ [28], mit dem Feststoff/Flüssigkeits-Gemische in einen Druckraum eingeschleust werden. Es besteht aus einem Hochdruck- und einem Niederdruckkreislauf, die beide durch einen Rotor verbunden sind. Dieser ist ein Drehventil, welches vier voneinander getrennte Kanäle enthält, die sich diametral durch einen Metallzylinder ausbreiten.

Die hydraulischen Systeme sind für sehr hohe Drücke bis zu 200 bar und Massenströme von mehreren $100 \mathrm{t} / \mathrm{h}$ geeignet. Ihre Vorteile sind:

- absolute Gasdichtheit zwischen den Räumen unterschiedlichen Druckes,

- es können alle Schüttgüter, auch stark schleißende oder kohäsive, verarbeitet werden,

- beliebige Korngrößen können verpumpt werden, allerdings muß bei anschließender Fest/Flüssig-Trennung eine Mindestkorngröße für das Trenngerät vorliegen,

- der Gutstrom ist voll kontinuierlich,

- die Technik ist erprobt, d. h. der Aufbau der Systeme erfolgt aus häufig verwendeten Einzelteilen.

Nachteilig ist, daß das Gut angefeuchtet wird; dies schränkt die Anwendungsbreite ein.

\subsection{Stopfschnecken, Extruder}

Stopfschnecken sind im Gegensatz zu Schneckenförderern ganz mit Gut gefüllt (Abb. 14,15). Durch den Materialpfropf innerhalb der Schnecke wird das System abgedichtet. Bei Extrudern wird Gut durch die Querschnittsveränderung der Schneckenkanäle stark kompaktiert.

Vorteile von Schneckensystemen sind:

- die kleine Baugröße,

- der einfache Aufbau,

- die hohen überwindbaren Druckdifferenzen,

- die kontinuierliche Arbeitsweise,

- die Eignung der Schnecke für nahezu alle Schüttgüter und

- die recht hohe Durchsatzleistung.

Diese Systeme haben folgende Nachteile:

- sie haben einen hohen spezifischen Energieverbrauch,

- bei schleißenden Gütern tritt hoher Verschleiß auf und

- das Schüttgut wird kornbeansprucht durch die Reibung zwischen Schnecke und Gehäuse mit dem Gut; weiterhin wird das Gut bei Verwendung einer Extrudierdüse auch kompaktiert.

Eine Erhöhung der Schüttdichte durch Kompaktierung des 

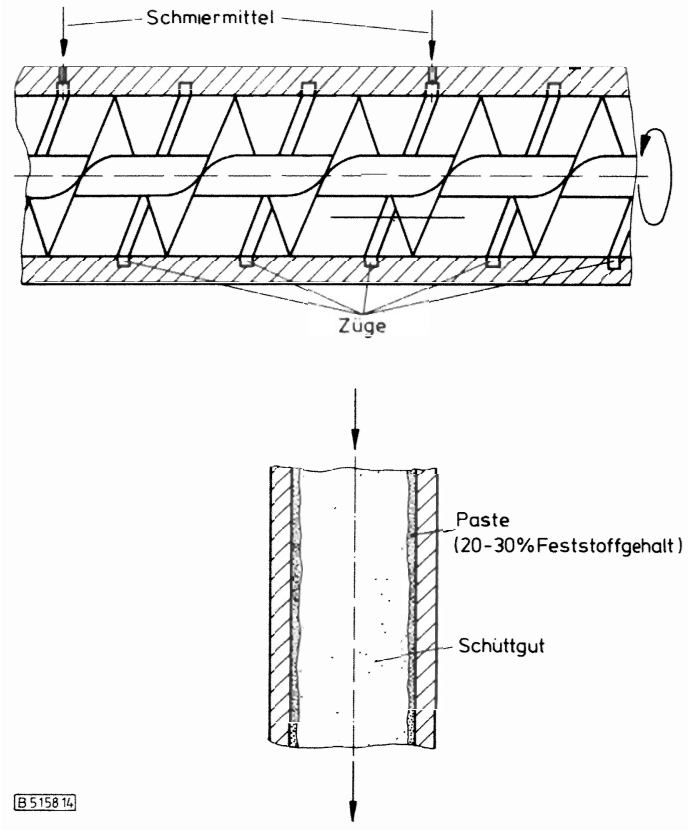

Abb. 14. Schnecke mit Zügen (oben) zur Schmiermittelzufuhr, um die Wandreibung wie im unten dargestellten Bild zu reduzieren (Inst. für MVM, Univ. K'he 1982); Einordnung: B III $1 / 2 / 1 / 2 / 2 / 1 / 1$ / $2 / 1 / 2 / 1 / 2$.
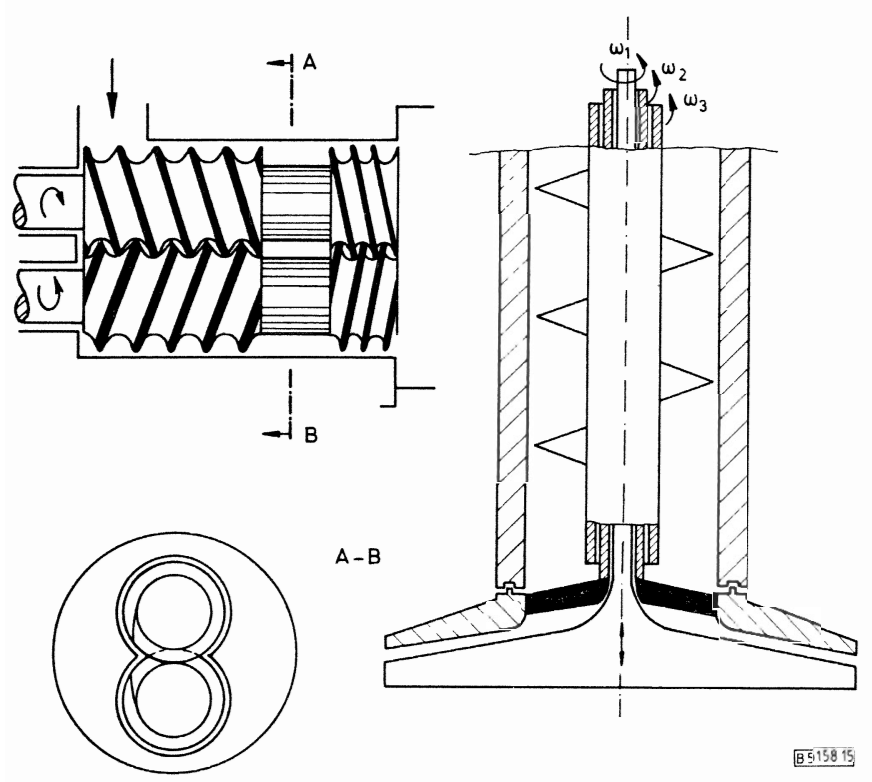

Abb. 15. (links) Zweischnecken-Verdrängersystem [29]: Einordnung: B III 1/1/1/2/2/1/2-3/2/1/2/1/1; (rechts) Stopfschnecke mit Zentrifugalaustrag (Inst. für MVM, Univ. K'he 1982); Einordnung: B IV $3 / 1 / 1 / 2 / 1 / 1 / 2-3 / 2 / 1 / 1 / 2 / 1$.

Gutes führt zu einer Anhebung der maximal möglichen Druckdifferenz. Die Kompaktierung wird durch Verkleinern des Gutvolumens in der Schnecke durch kontinuierliche Reduzierung des Gehäusedurchmessers oder durch Verdikkung des Schneckenkern-Durchmessers erzielt. Der Zweiwellenextruder ist ein reines Verdrängersystem.

Weiterhin existieren Schmelzextruder zur Verflüssigung von Kohle, Kunststoff und Granulaten, bei denen der Mantel und auch evtl. die Schnecke beheizt werden.

Extruder sind zur Überwindung extrem hoher Druckdifferenzen geeignet, z. B. bis zu ca. 1000 bar in der KunststoffIndustrie und für hohe Durchsätze bis zu mehreren $100 \mathrm{t} / \mathrm{h}$.
Der hohe spezifische Energieverbrauch von Extrudern sinkt bei formschlüssigen Zweiwellenextrudern auf ca. $1 / 3$ bis $1 / 4$ dessen von Einwellenextrudern ab.

\subsection{Kolbenförderer}

Plunger- bzw. Kolbenpumpen werden sehr häufig für den Transport von Suspensionen verwendet, z. B. beim Betontransport (Abb. 16). Solche Systeme finden auch in der Kohleverflüssigung Anwendung.

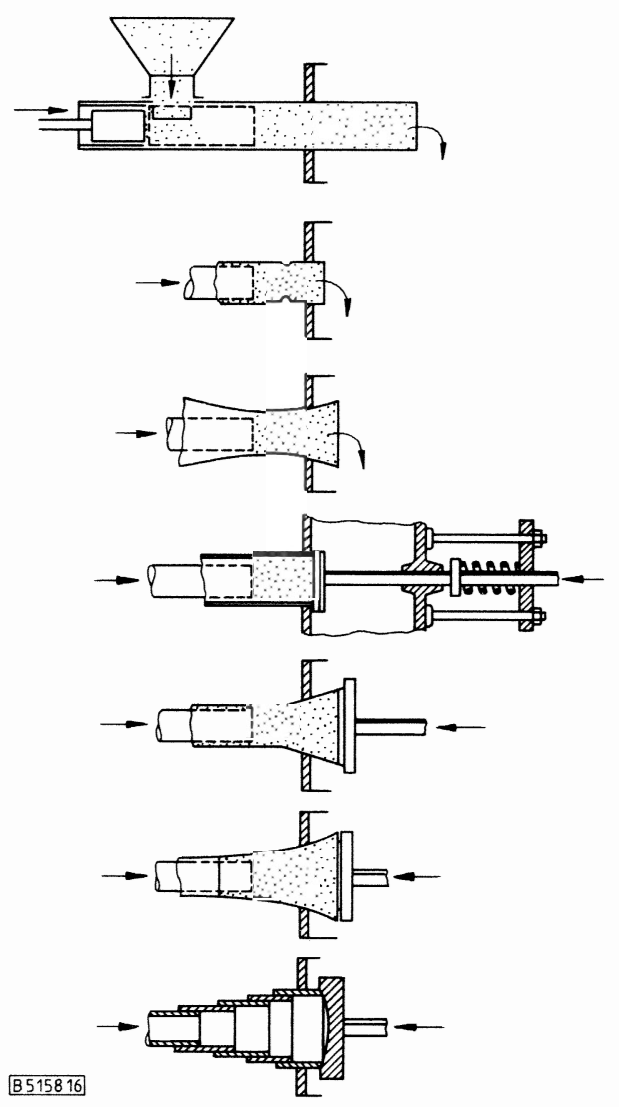

Abb. 16. Kolbenförderer mit Drehschieber und verschiedenen Formstïcken sowie Verschlußklappen [30]; Einordnung: B V $1 / 1 / 3 / 3 / 1 / 1 / 1 / 3 / 1 / 1 / 1 / 1$.

Plungerpumpen eignen sich zum kontinuierlichen Einschleusen von Feststoff/Flüssigkeits-Gemischen in Druckprozesse. Die möglichen Drücke erreichen weit über 100 bar, und die Korndurchmesser können über $20 \mathrm{~mm}$ liegen [15]. Sie haben sich schon in vielen Einsatzgebieten bewährt.

Außer für Suspensionen sind die Kolbenpumpen auch als reine Schüttgutpumpen einzusetzen, wie z. B. als Version mit einem Drehschieberventil oder als Strangpresse, bei der die Dichtwirkung auf der Kompaktierung des Gutes beruht. Es kann in diesen Systemen jedes Material verwendet werden, da es durch eine Schnecke zugeführt werden kann und nach der Kompaktierung durch einen Schälkopf wieder aufgelokkert werden kann. Eine Abdichtung dieses Systems erfolgt beim An- oder Abfahren mit Hilfe eines Ventils. Der Massendurchsatz dieser Systeme kann bis zu $10 \mathrm{t} / \mathrm{h}$ betragen. Vorteile der Systeme:

- geringe Abhängigkeit von der Fließfähigkeit des Gutes,

- sehr hohe Differenzdrücke sind möglich und

- eine vollständige Gasabdichtung ist einfach zu realisieren,

- nahezu alle Korngrößen können verarbeitet werden (Ausnahme: Feinstgut, wegen zu hohem Verschleiß).

Nachteile des Kolbenförderers: 


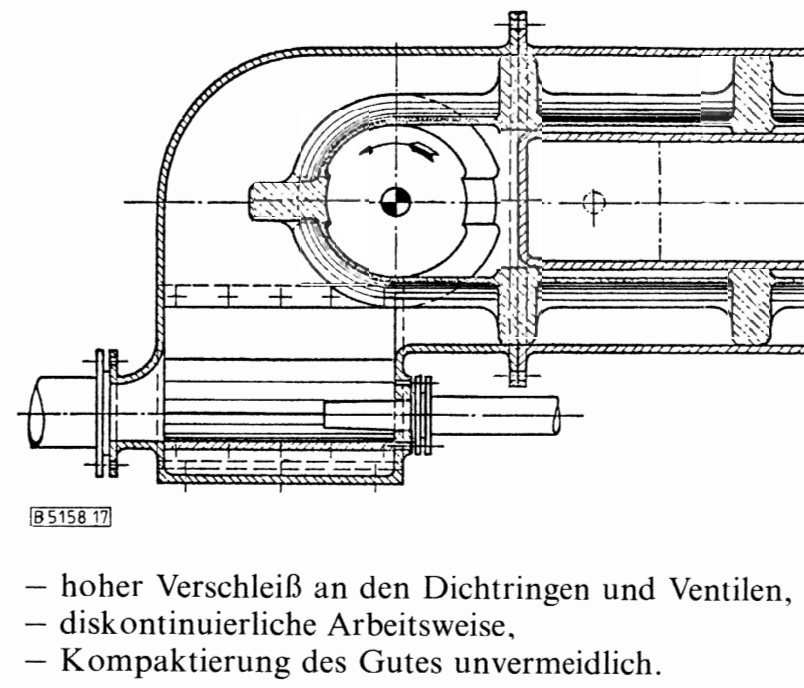

\subsection{Gliederkettenförderer}

Dieses System (Abb. 17) besteht aus einer Vielzahl von scheibenförmigen Kolben, die durch eine Kette oder Kolbenstange miteinander verbunden sind. Über ein Antriebsrad und ein Umlenkrad wird eine lineare Bewegung erzeugt. Die Kette durchläuft dabei im Vorlauf und Rücklauf jeweils ein Rohr. Zwei auf der Kette montierte Kolben und die stationäre Rohrwand bilden eine Schleusenkammer.

Höhere Drücke können nur mit Systemen überwunden werden, bei denen die Wände des Rohres mit einer aufblasbaren Membran ausgekleidet sind und ein Druckausgleich vorgesehen ist.

Antriebsenergie ist praktisch nur zur Überwindung der Reibung erforderlich, da an den Kolben nahezu Druckausgleich herrscht.

Vorteile:

- hohe Druckdifferenzen sind möglich,

- alle Korngrößen sind einsetzbar,

- es tritt keine Kornbeanspruchung auf,

- geringer spezifischer Energieverbrauch.

Nachteile:

- sehr aufwendige, platzbeanspruchende Bauweise,

- sehr hoher Verschleiß.

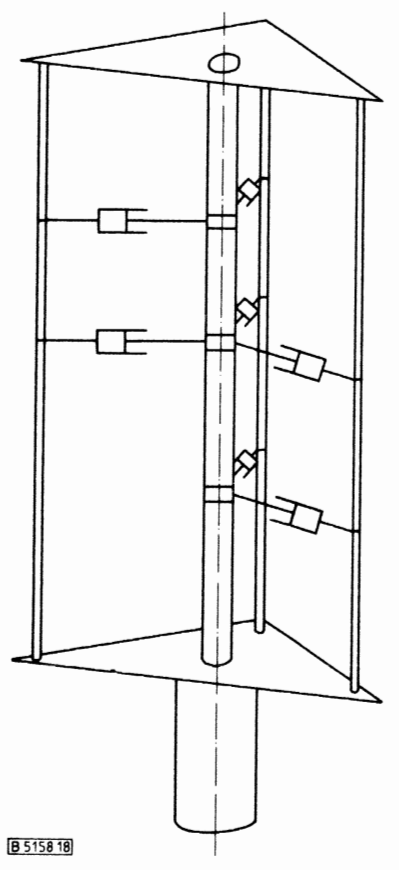

Abb. 18. Schematische Darstellung des Systems ,flexibler Schlauch mit Selbsthemmung".
Abb. 17. Gliederbandförderer [31]; Einordnung: B VI 3/2/2/1/ $2 / 2 / 1 / 3 / 2 / 2 / 1 / 1$

\section{Schlußbemerkung zur Studie}

In der ungekürzten Fassung dieser Arbeit wurden 231 Patente und 247 Veröffentlichungen gesammelt und bearbeitet [33].

Allein die Zahl und Vielfalt der vorgeschlagenen Systeme zeigt, daß es ,die Patentlösung“ bis heute nicht gibt; eine einzige, universell verwendbare Konstruktion ist bei der Vielgestaltigkeit der Aufgabenstellungen und dem Verhalten der verarbeiteten Produkte auch kaum zu erwarten.

Anhand der Beurteilung der einzelnen aufgezeigten Teillösungen lassen sich die für das Problem des jeweiligen Anwenders am besten geeigneten Systeme aussuchen.

Eine Schwierigkeit liegt allerdings darin, daß es augenblicklich noch keine hinreichende Kennzeichnung und Untersuchungsmöglichkeit des Verhaltens der Schüttgutpackung bei Be- oder Entgasung sowie unter hohem Druckgefälle und des Verhaltens beim Fließen in dem entsprechenden Schleussystem gibt.

Deshalb sind bei der Auslegung einer Anlage weiterhin aufwendige Experimente unerläßlich.

\section{Anwendungsbeispiel}

Für einen uns interessierenden Anwendungsfall, den kontinuierlichen Austrag von Filterkuchen aus einem Druck filter, sollte ein Schleussystem erarbeitet werden, das folgende Forderungen erfüllt :

- es muß im Rahmen der Schwankung der Produkteigenschaften funktionssicher sein,

- es sollte für unterschiedliche Produkte einsetzbar sein;

- der Gasverlust sollte minimal sein und

- es sollte einfach aufzubauen sein.

Durch systematisches Vorgehen wurde ein System nach dem Dichtprinzip der Gassperrstrecke, ähnlich den Abb. 9, 10 und 11, entwickelt (s. Abb. 18).

Das dichtende Bauteil ist ein mit Gut gefüllter Schlauch, wie er serienmäßig für Betonpumpen verwendet wird (bis ca. 6 bar druckdicht); er ist als billiges Verschleißteil sehr einfach auszutauschen. Der Schlauch ist in seinem Durchmesser/ Längen-Verhältnis so dimensioniert, daß Selbsthemmung des Gutes auftritt. Ein Fließen des Gutes wird durch eine hydraulisch gesteuerte geringe Querbewegung des Schlauches bewirkt. Die Auslaufmenge wird durch eine drehende Dichtscheibe am unteren Rohrende reguliert. Die Bewegung der Dichtscheibe und das Bewegen des Schlauches werden durch berührungsloses Messen des Feststoffspiegels gesteuert. In Vorversuchen erwies sich das System für zwei Produkte sehr unterschiedlichen Fließverhaltens als geeignet.

Durchströmungsversuche an einem Modell ergaben die in 


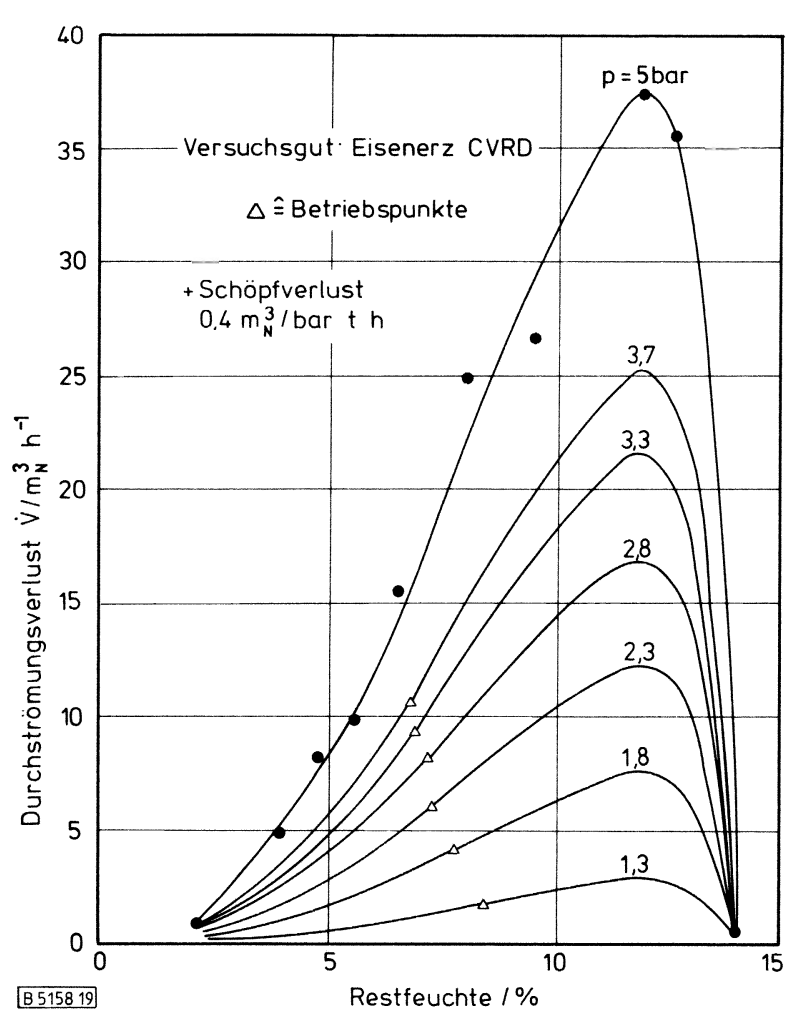

Abb. 19. Durchströmungsverlust des Schleussystems „flexibles Rohr mit Selbsthemmung “. (Die Betriebspunkte sind erreichte Restfeuchten einer hyperbaren Vakuumfiltrations-Anlage im Institut.)

Abb. 19 dargestellten Leckgasströme für ein geplantes Schleussystem mit einer Querschnittsfläche von $80 \mathrm{~cm}^{2}$ und einer Schlauchlänge von $2 \mathrm{~m}$. Die Leckverluste betragen nur einen Bruchteil derer einer Zellenradschleuse mit gleichem Massendurchsatz, die außerdem nur bis ca. 1,5 bar einsetzbar ist.

Dieses Beispiel zeigt eine universell einsetzbare kostengünstige Lösung eines Schleusproblems durch systematische Vorgehensweise.

Eingegangen am 15. Dezember 1983 [B 5158]

\section{Literatur}

[1] Winnacker/Küchler: Chemische Technologie. Organische Technologie I, 4. Aufl., Carl Hanser Verlag, München 1981.

[2] Lehrbuch der technischen Chemie. Technische Hochschule für Chemie, Leuna-Merseburg, VEB Deutscher Verlag für Grundstoffindustrie 1974.

[3] Winnacker/Küchler: Chemische Technologie. Organische Technologie III, 3. Aufl., Carl Hanser Verlag, München 1972.
[4] Schormüller, J.: Lehrbuch der Lebensmittelchemie, 2. Aufl., Springer Verlag, Berlin 1974

[5] Loncin, M.: Die Grundlagen der Verfahrenstechnik in der Lebensmittelindustrie, Verlag Sauerländer, Aarau 1969.

[6] Pahl/Beitz: Konstruktionslehre, Springer Verlag, Berlin 1977.

[7] VDI-Richtlinien 2222 Blatt 2. Konstruktionsmethodik. Erstellung und Anwendung von Konstruktionskatalogen, Juli 1977.

[8] Molerus, O.: Fluid-Feststoff-Strömungen, Springer Verlag, Berlin 1982.

[9] Gupte, A. R.: Dissertation, Univ. Karlsruhe 1970.

[10] Carman, P. C.: Flow of Gases Through Porous Media, Butterworth, London 1956.

[11] Kozeny, J.: Sitzungsber. Akad. Wiss. Wien, Math.-Naturwiss. Kl., Abt. 2 A 136, 271 (1927).

[12] Stahl, W.: Hochschulkurs „Fest-Flüssig-Trennung“, Univ. Karlsruhe 1981.

[13] Rumpf, H.: Mechanische Verfahrenstechnik, Carl Hanser Verlag, München 1975.

[14] Schwedes, J.: Fließverhalten von Schüttgütern in Bunkern, Verlag Chemie, Weinheim 1968.

[15] Reimert, R.: Chem.-Ing.-Tech. 53 (1981) Nr. 5, S. 335/344.

[16] Dt. Pat. Schrift Nr. 862 429, pat.28.7.51 (Anmelder: J. Möller, Hamburg).

[17] Dt. Pat. Schrift Nr. 843 090, pat. 2. 10. 48 (Anmelder: Metallgesellschaft AG, Frankfurt/M.).

[18] Dt. Anm. Schrift Nr. 1013 575, angem. 10.9. 55 (Anmelder: E. Harvengt, Belgien).

[19] Ahland, E.; Schönlebe, K.: Chem.-Ing.-Tech. 51 (1979) Nr. 3, S. $216 / 217$.

[20] Albright, C. W.; Holden, J. H.; Simons, H. P.; Schmidt, L. D.: Ind. Eng. Chem. 43 (1951) Nr. 8, S. 1837/1840.

[21] Albright, C. W.: Dissertation, West Virginia University 1953.

[22] Leung, L. S.; Wilson, L. A.: Powder Technol. 7 (1973) S. $343 / 349$

[23] Leung, L. S.: Cocurrent downflow o suspension in standpipes. Proc. Int. Fluidization Conf., organized by Engineering Foundation, 1975.

[24] Bonin, J. H.; Cantey, D. E.; Daniel, A. D.; Meyer, J. W.: Development of dry coal feeders. Proc. Conf. on Coal Feeding Systems, Pasadena 1977, S. 195/239.

[25] Harding, J. C.: Foster-Miller's development of dry coal feed systems. Proc. Conf. on Coal Feeding Systems, Pasadena 1977, S. $285 / 323$.

[26] Hobday, J. M.: Development of coal-feeding systems at the morgantown energy research center. Proc. Conf. on Coal Feeding Systems, Pasadena 1977, S. 100/133.

[27] Weber, M.: Strömungsfördertechnik, Krausskopf-Verlag, Mainz 1974.

[28] Dt. Offenl. Schrift Nr. 2503 400, angem. 28. 1.75 (Anmelder: Kamyr Inc., Clenns Falls, N.Y., USA).

[29] Dt. Offenl. Schrift Nr. 1442746 , angem. 27. 11. 63 (Anmelder: Kalle KG, Wiesbaden).

[30] US-Pat. Schrift Nr. 2906417 , angem. 22. 9. 52 (Anmelder: G. Rossi, Mailand).

[31] Dt. Pat. Schrift Nr. 593844, pat. 15. 2. 34 (Anmelder: G. Geldmacher, Dortmund).

[32] Fest-Flüssig-Trennung, Hochschulkurs 1983. Institut für MVM, Univ. Karlsruhe.

[33] GVT-Bericht 4940 „Ein- und Austrag von Schüttgütern in und aus Druckräumen“" Institut für MVM (W. Stahl, R. Stadler), Univ. Karlsruhe 1982. 